Acta Crystallographica Section E

Structure Reports

Online

ISSN 1600-5368

\section{Hazel S. Wilkinson and William T. A. Harrison*}

Department of Chemistry, University of Aberdeen, Meston Walk, Aberdeen AB24 3UE, Scotland

Correspondence e-mail:

w.harrison@abdn.ac.uk

\section{Key indicators}

Single-crystal X-ray study

$T=120 \mathrm{~K}$

Mean $\sigma(\mathrm{C}-\mathrm{C})=0.002 \AA$

$R$ factor $=0.019$

$w R$ factor $=0.049$

Data-to-parameter ratio $=17.6$

For details of how these key indicators were automatically derived from the article, see http://journals.iucr.org/e.

\title{
Piperazinium hydrogenarsenate monohydrate
}

In the title compound, $\mathrm{C}_{4} \mathrm{H}_{12} \mathrm{~N}_{2}{ }^{2+} \cdot \mathrm{HAsO}_{4}{ }^{2-} \cdot \mathrm{H}_{2} \mathrm{O}$, the component species interact by way of $\mathrm{N}-\mathrm{H} \cdots \mathrm{O}$ and $\mathrm{O}-\mathrm{H} \cdots \mathrm{O}$ hydrogen bonds, the latter leading to infinite sheets of $\mathrm{HAsO}_{4}{ }^{2-}$ anions and water molecules containing $R_{6}^{6}(18)$ loops. The asymmetric unit contains one anion, one water molecule and half each of two centrosymmetric cations.

\section{Comment}

The $\mathrm{As}^{\mathrm{V}}$-containing title compound, (I), (Fig. 1), arose unexpectedly as a result of atmospheric oxidation from a solutionmediated reaction containing $\mathrm{As}^{\mathrm{III}}$ (Lee \& Harrison, 2004). It complements $\mathrm{C}_{4} \mathrm{H}_{12} \mathrm{~N}_{2} \cdot 2 \mathrm{H}_{2} \mathrm{AsO}_{4}$, (II) (Wilkinson \& Harrison, 2007), which contains the same organic cation accompanied by monovalent dihydrogenarsenate anions. Compound (I) is isostructural with its hydrogenphosphate analogue (Riou et al., 1993).<smiles>O=[SH]OC1C[NH2+]CC[NH2+]1</smiles>

(I)

The tetrahedral $\mathrm{HAsO}_{4}{ }^{2-}$ anion in (I) shows three short As-O links with formal partial double-bond character, and one longer $\mathrm{As}-\mathrm{OH}$ bond (Table 1). The mean As-O bond

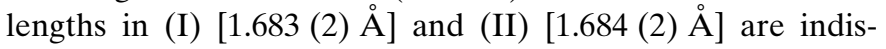
tinguishable.

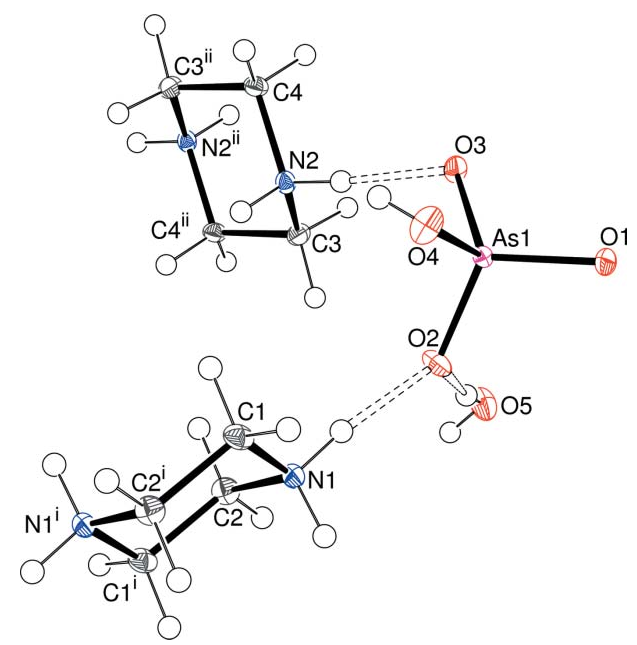

Figure 1

The molecular structure of (I), with the atom-numbering scheme. Displacement ellipsoids are drawn at the $50 \%$ probability level and $\mathrm{H}$ atoms are showwn as spheres of arbitrary radius. Hydrogen bonds are indicated by double-dashed lines. [Symmetry codes: (i) $-x, 1-y, 1-z$; (ii) $-x, 2-y, 1-z$.]
Received 14 February 2007 Accepted 15 February 2007 


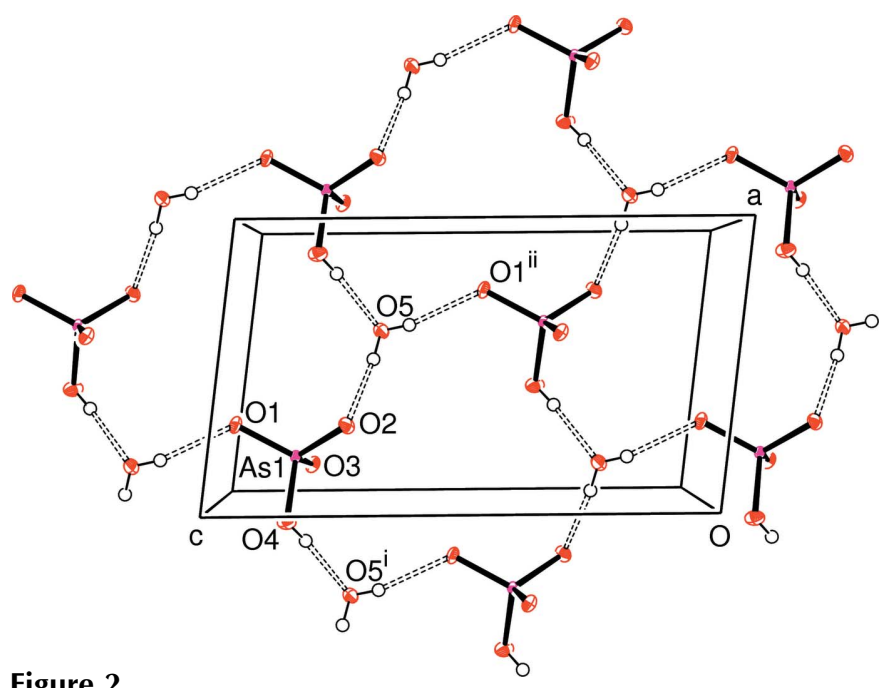

Figure 2

Detail of a part of an (010) hydrogen-bonded sheet of $\mathrm{HAsO}_{4}{ }^{2-}$ groups and water molecules in (I), with $\mathrm{H}$ bonds indicated by double-dashed lines. (Symmetry codes as in Table 1.)

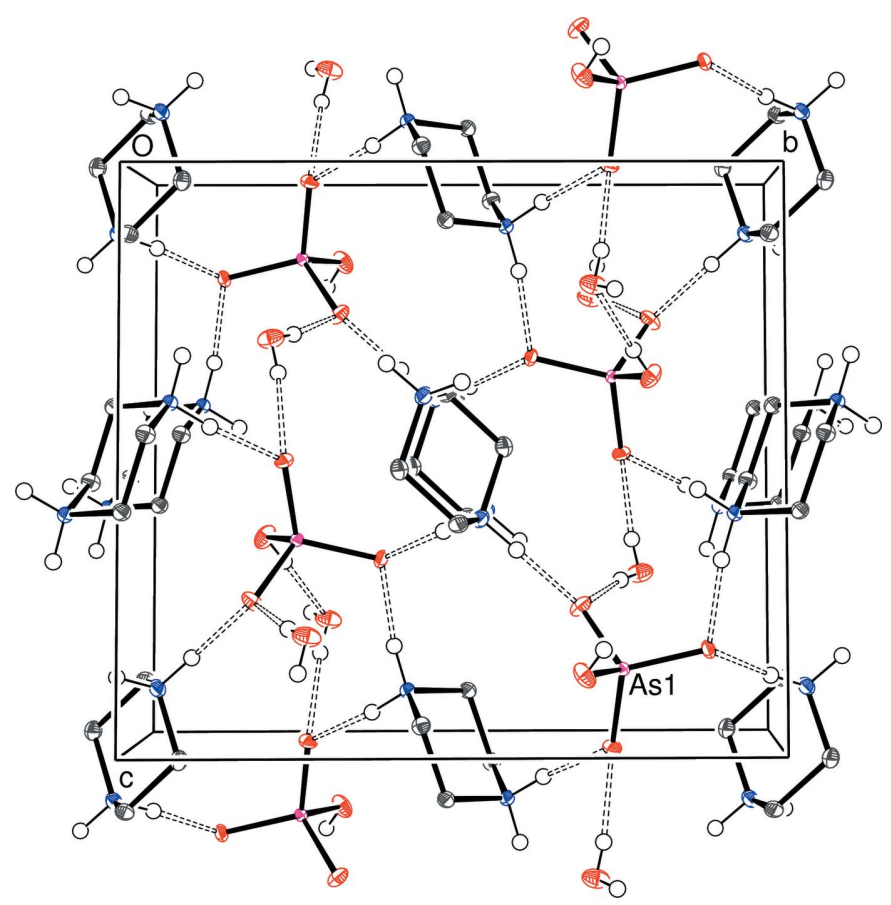

Figure 3

A view down [100] of the unit-cell packing in (I), showing the (010) hydrogenarsenate-water layers mediated by the organic cations. Hydrogen bonds are indicated by double-dashed lines and C-bound $\mathrm{H}$ atoms have been omitted for clarity.

The asymmetric unit contains one anion, one water molecule and half each of two centrosymmetric cations. Each cation adopts a typical chair conformation.

As well as Coulombic forces, the component species in (I) interact by way of a network of $\mathrm{O}-\mathrm{H} \cdots \mathrm{O}$ and $\mathrm{N}-\mathrm{H} \cdots \mathrm{O}$ hydrogen bonds (Table 2). The $\mathrm{HAsO}_{4}{ }^{2-}$ dianions and water molecules are linked into infinite sheets (Fig. 2) propagating in (010) by way of the $\mathrm{O}-\mathrm{H} \cdots \mathrm{O}$ bonds. The water molecule accepts one hydrogen bond and makes two hydrogen bonds. Unlike the case in many related molecular salts (Lee \&

Harrison, 2003), there are no direct hydrogen-bond links between hydrogenarsenate groups. A supramolecular $R_{6}^{6}(18)$ loop (Bernstein et al., 1995) arises from this hydrogen-bond topology.

The hydrogenarsenate-water sheets are bridged by the piperazinium cations, which participate in two strong $\mathrm{N}-$ $\mathrm{H} \cdots \mathrm{O}$ interactions from each of their $\mathrm{NH}_{2}$ groups to $\mathrm{O}$ atoms of nearby hydrogenarsenate tetrahedra. Thus, there are no hydrogen-bond links between organic cations and water molecules in (I). Overall, a layered architecture (Fig. 3) results, in which layers of organic and inorganic species alternate along [010]. Compound (II) also possesses alternating inorganic and organic layers; in this compound, supramolecular $R_{6}^{6}(24)$ loops arise for each circuit of six $\mathrm{H}_{2} \mathrm{AsO}_{4}{ }^{-}$tetrahedra within a sheet.

\section{Experimental}

In an attempt to synthesize an analogue of $\left(\mathrm{H}_{3} \mathrm{NCH}_{2} \mathrm{CH}_{2} \mathrm{NH}_{3}\right)$ $\left[\mathrm{AsO}_{2}\right]_{2}$ (Lee \& Harrison, 2004), aqueous solutions of piperazine (0.1 $M)$ and $\mathrm{As}_{2}{ }_{2} \mathrm{O}_{3}(0.1 \mathrm{M})$ were mixed, resulting in a colourless mixture. Translucent faceted truncated cubes of $\mathrm{As}_{2} \mathrm{O}_{3}$ recrystallized after one day. After several months, colourless slabs of (I) were dredged from the viscous liquor.

Crystal data

$\mathrm{C}_{4} \mathrm{H}_{12} \mathrm{~N}_{2}{ }^{2+} \cdot \mathrm{HAsO}_{4}{ }^{2-} \cdot \mathrm{H}_{2} \mathrm{O}$

$M_{r}=246.10$

Monoclinic, $P 2_{1} / n$

$a=6.5093(2) \mathrm{A}$

$b=12.5329(3) \AA$

$c=11.2873(3) \AA$

$\beta=97.0816(16)^{\circ}$

\section{Data collection}

Nonius KappaCCD area-detector diffractometer

Absorption correction: multi-scan (SADABS; Bruker, 2003)

$T_{\min }=0.318, T_{\max }=0.625$

\section{Refinement}

$R\left[F^{2}>2 \sigma\left(F^{2}\right)\right]=0.019$

$w R\left(F^{2}\right)=0.049$

$S=1.09$

2094 reflections

119 parameters

Table 1

Selected bond lengths ( $\mathrm{A})$.

\begin{tabular}{llll}
\hline As1-O1 & $1.6627(12)$ & As1-O3 & $1.6760(11)$ \\
As1-O2 & $1.6680(12)$ & As1-O4 & $1.7264(12)$ \\
\hline
\end{tabular}

Table 2

Hydrogen-bond geometry $\left(\AA{ }^{\circ}\right)$.

\begin{tabular}{lllll}
\hline$D-\mathrm{H} \cdots A$ & $D-\mathrm{H}$ & $\mathrm{H} \cdots A$ & $D \cdots A$ & $D-\mathrm{H} \cdots A$ \\
\hline $\mathrm{O} 4-\mathrm{H} 1 \cdots \mathrm{O} 5^{\mathrm{i}}$ & $0.842(10)$ & $1.812(10)$ & $2.6492(19)$ & $173(2)$ \\
$\mathrm{N} 1-\mathrm{H} 1 C \cdots \mathrm{O} 2$ & 0.92 & 1.73 & $2.6482(18)$ & 171 \\
$\mathrm{~N} 1-\mathrm{H} 1 D \cdots \mathrm{O} 3^{\text {ii }}$ & 0.92 & 1.76 & $2.6722(18)$ & 173 \\
$\mathrm{~N} 2-\mathrm{H} 2 C \cdots \mathrm{O} 1^{\text {iii }}$ & 0.92 & 1.77 & $2.6788(18)$ & 169
\end{tabular}

15415 measured reflections 2094 independent reflections 1898 reflections with $I>2 \sigma(I)$ $R_{\text {int }}=0.029$ atoms treated by a mixture of independent and constrained

$\Delta \rho_{\max }=0.46{\mathrm{e} \AA^{-3}}^{-3}$

$\Delta \rho_{\min }=-0.47{\mathrm{e} \AA^{-3}}^{-3}$ 


\begin{tabular}{lllll}
$D-\mathrm{H} \cdots A$ & $D-\mathrm{H}$ & $\mathrm{H} \cdots A$ & $D \cdots A$ & $D-\mathrm{H} \cdots A$ \\
\hline $\mathrm{N} 2-\mathrm{H} 2 D \cdots \mathrm{O} 3$ & 0.92 & 1.74 & $2.6548(18)$ & 172 \\
$\mathrm{O} 5-\mathrm{H} 2 \cdots \mathrm{O} 2$ & $0.842(10)$ & $1.863(10)$ & $2.6998(18)$ & $173(2)$ \\
$\mathrm{O} 5-\mathrm{H} 3 \cdots \mathrm{O} 1^{\text {iv }}$ & $0.836(10)$ & $1.892(12)$ & $2.7065(18)$ & $164(2)$ \\
\hline
\end{tabular}

Symmetry codes: (i) $x-1, y, z$; (ii) $-x+\frac{1}{2}, y-\frac{1}{2},-z+\frac{3}{2}$; (iii) $x-\frac{1}{2},-y+\frac{3}{2}, z-\frac{1}{2}$; (iv) $x+\frac{1}{2},-y+\frac{3}{2}, z-\frac{1}{2}$.

O-bound $\mathrm{H}$ atoms were found in difference maps and their positions were refined with the restraint $\mathrm{O}-\mathrm{H}=0.85$ (1) $\AA$. C- and Nbonded $\mathrm{H}$ atoms were positioned geometrically, with $\mathrm{C}-\mathrm{H}=0.99 \AA$ and $\mathrm{N}-\mathrm{H}=0.92 \AA$, and refined as riding atoms. $U_{\text {iso }}(\mathrm{H})=$ $1.2 U_{\text {eq }}$ (carrier) for all $\mathrm{H}$ atoms.

Data collection: COLLECT (Nonius, 1998); cell refinement: SCALEPACK (Otwinowski \& Minor, 1997); data reduction: SCALEPACK and DENZO (Otwinowski \& Minor, 1997), and SORTAV (Blessing, 1995); program(s) used to solve structure: SHELXS97 (Sheldrick, 1997); program(s) used to refine structure: SHELXL97 (Sheldrick, 1997); molecular graphics: ORTEP-3 (Farrugia, 1997); software used to prepare material for publication: SHELXL97.
HSW thanks the Carnegie Trust for the Universities of Scotland for an undergraduate vacation studentship.

\section{References}

Bernstein, J., Davis, R. E., Shimoni, L. \& Chang, N.-L. (1995). Angew. Chem. Int. Ed. Engl. 34, 1555-1573.

Blessing, R. H. (1995). Acta Cryst. A51, 33-38.

Bruker (2003). SADABS. Bruker AXS Inc., Madison, Wisconsin, USA.

Farrugia, L. J. (1997). J. Appl. Cryst. 30, 565.

Lee, C. \& Harrison, W. T. A. (2003). Acta Cryst. E59, m1151-m1153.

Lee, C. \& Harrison, W. T. A. (2004). Acta Cryst. C60, m215-m218.

Nonius (1998). COLLECT. Nonius BV, Delft, The Netherlands.

Otwinowski, Z. \& Minor, W. (1997). Methods in Enzymology, Vol. 276, Macromolecular Crystallography, Part A, edited by C. W. Carter Jr \& R. M. Sweet, pp. 307-326. New York: Academic Press.

Riou, D., Loiseau, T. \& Ferey, G. (1993). Acta Cryst. C49, 1237-1238.

Sheldrick, G. M. (1997). SHELXS97 and SHELXL97. University of Göttingen, Germany.

Wilkinson, H. S. \& Harrison, W. T. A. (2007). Acta Cryst. E63, m26-m28. 


\section{supporting information}

Acta Cryst. (2007). E63, m905-m907 [https://doi.org/10.1107/S1600536807007763]

\section{Piperazinium hydrogenarsenate monohydrate}

\section{Hazel S. Wilkinson and William T. A. Harrison}

Piperazinium hydrogenarsenate monohydrate

Crystal data

$\mathrm{C}_{4} \mathrm{H}_{12} \mathrm{~N}_{2}{ }^{2+} \cdot \mathrm{HAsO}_{4}{ }^{2-} \cdot \mathrm{H}_{2} \mathrm{O}$

$M_{r}=246.10$

Monoclinic, $P 2_{1} / n$

Hall symbol: -P $2 \mathrm{yn}$

$a=6.5093(2) \AA$

$b=12.5329(3) \AA$

$c=11.2873(3) \AA$

$\beta=97.0816(16)^{\circ}$

$V=913.80(4) \AA^{3}$

$Z=4$

\section{Data collection}

Nonius KappaCCD area-detector diffractometer

Radiation source: fine-focus sealed tube Graphite monochromator $\omega$ and $\varphi$ scans

Absorption correction: multi-scan

(SADABS; Bruker, 1999)

$T_{\min }=0.318, T_{\max }=0.625$

\section{Refinement}

Refinement on $F^{2}$

Least-squares matrix: full

$R\left[F^{2}>2 \sigma\left(F^{2}\right)\right]=0.019$

$w R\left(F^{2}\right)=0.049$

$S=1.09$

2094 reflections

119 parameters

3 restraints

Primary atom site location: structure-invariant direct methods

Secondary atom site location: difference Fourier map
$F(000)=504$

$D_{\mathrm{x}}=1.789 \mathrm{Mg} \mathrm{m}^{-3}$

Mo $K \alpha$ radiation, $\lambda=0.71073 \AA$

Cell parameters from 2149 reflections

$\theta=2.9-27.5^{\circ}$

$\mu=3.71 \mathrm{~mm}^{-1}$

$T=120 \mathrm{~K}$

Slab, colourless

$0.40 \times 0.28 \times 0.14 \mathrm{~mm}$

15415 measured reflections

2094 independent reflections

1898 reflections with $I>2 \sigma(I)$

$R_{\text {int }}=0.029$

$\theta_{\max }=27.5^{\circ}, \theta_{\min }=3.6^{\circ}$

$h=-8 \rightarrow 8$

$k=-16 \rightarrow 16$

$l=-14 \rightarrow 14$

Hydrogen site location: inferred from neighbouring sites

$\mathrm{H}$ atoms treated by a mixture of independent and constrained refinement

$w=1 /\left[\sigma^{2}\left(F_{\mathrm{o}}^{2}\right)+(0.0187 P)^{2}+0.7794 P\right]$ where $P=\left(F_{\mathrm{o}}^{2}+2 F_{\mathrm{c}}^{2}\right) / 3$

$(\Delta / \sigma)_{\max }=0.001$

$\Delta \rho_{\max }=0.46 \mathrm{e} \AA^{-3}$

$\Delta \rho_{\min }=-0.47$ e $\AA^{-3}$

Extinction correction: SHELXL97 (Sheldrick, 1997), $\mathrm{Fc}^{*}=\mathrm{kFc}\left[1+0.001 \mathrm{xFc}^{2} \lambda^{3} / \sin (2 \theta)\right]^{-1 / 4}$

Extinction coefficient: 0.0104 (7) 


\section{Special details}

Geometry. All e.s.d.'s (except the e.s.d. in the dihedral angle between two 1.s. planes) are estimated using the full covariance matrix. The cell e.s.d.'s are taken into account individually in the estimation of e.s.d.'s in distances, angles and torsion angles; correlations between e.s.d.'s in cell parameters are only used when they are defined by crystal symmetry. An approximate (isotropic) treatment of cell e.s.d.'s is used for estimating e.s.d.'s involving 1.s. planes.

Refinement. Refinement of $F^{2}$ against ALL reflections. The weighted $R$-factor $w R$ and goodness of fit $S$ are based on $F^{2}$, conventional $R$-factors $R$ are based on $F$, with $F$ set to zero for negative $F^{2}$. The threshold expression of $F^{2}>\sigma\left(F^{2}\right)$ is used only for calculating $R$-factors (gt) etc. and is not relevant to the choice of reflections for refinement. $R$-factors based on $F^{2}$ are statistically about twice as large as those based on $F$, and $R$ - factors based on ALL data will be even larger.

Fractional atomic coordinates and isotropic or equivalent isotropic displacement parameters $\left(\hat{A}^{2}\right)$

\begin{tabular}{lllll}
\hline & $x$ & $y$ & $z$ & $U_{\text {iso }}{ }^{*} U_{\text {eq }}$ \\
\hline As1 & $0.14908(2)$ & $0.754902(12)$ & $0.858124(14)$ & $0.00805(8)$ \\
O1 & $0.2714(2)$ & $0.73938(9)$ & $0.99528(11)$ & $0.0136(3)$ \\
O2 & $0.26775(19)$ & $0.69220(10)$ & $0.75530(11)$ & $0.0159(3)$ \\
O3 & $0.10970(18)$ & $0.88403(9)$ & $0.82395(10)$ & $0.0124(2)$ \\
O4 & $-0.09090(19)$ & $0.69654(10)$ & $0.85928(12)$ & $0.0179(3)$ \\
H1 & $-0.175(3)$ & $0.7282(16)$ & $0.8085(17)$ & $0.021^{*}$ \\
C1 & $-0.0938(3)$ & $0.51032(14)$ & $0.60902(14)$ & $0.0139(4)$ \\
H1A & -0.1834 & 0.5738 & 0.5930 & $0.017^{*}$ \\
H1B & -0.1097 & 0.4841 & 0.6901 & $0.017^{*}$ \\
C2 & $0.1611(3)$ & $0.57581(13)$ & $0.48176(15)$ & $0.0141(3)$ \\
H2A & 0.3098 & 0.5916 & 0.4806 & $0.017^{*}$ \\
H2B & 0.0818 & 0.6419 & 0.4607 & $0.017^{*}$ \\
N1 & $0.1258(2)$ & $0.54061(11)$ & $0.60339(13)$ & $0.0133(3)$ \\
H1C & 0.1617 & 0.5949 & 0.6568 & $0.016^{*}$ \\
H1D & 0.2096 & 0.4831 & 0.6255 & $0.016^{*}$ \\
N2 & $0.0015(2)$ & $0.92247(11)$ & $0.59340(12)$ & $0.0105(3)$ \\
H2C & -0.0643 & 0.8658 & 0.5532 & $0.013^{*}$ \\
H2D & 0.0352 & 0.9027 & 0.6719 & $0.013^{*}$ \\
C3 & $0.1939(3)$ & $0.94754(13)$ & $0.54065(15)$ & $0.0124(3)$ \\
H3A & 0.2824 & 0.8832 & 0.5426 & $0.015^{*}$ \\
H3B & 0.2720 & 1.0040 & 0.5883 & $0.015^{*}$ \\
C4 & $-0.1414(3)$ & $1.01496(13)$ & $0.58730(15)$ & $0.0119(3)$ \\
H4A & -0.0759 & 1.0740 & 0.6366 & $0.014^{*}$ \\
H4B & -0.2701 & 0.9944 & 0.6200 & $0.014^{*}$ \\
O5 & $0.6199(2)$ & $0.78709(11)$ & $0.70613(12)$ & $0.0190(3)$ \\
H2 & $0.509(2)$ & $0.7551(15)$ & $0.715(2)$ & $0.023^{*}$ \\
H3 & $0.645(4)$ & $0.7727(17)$ & $0.6371(11)$ & $0.023^{*}$ \\
& & & &
\end{tabular}

Atomic displacement parameters $\left(\AA^{2}\right)$

\begin{tabular}{lllllll}
\hline & $U^{11}$ & $U^{22}$ & $U^{33}$ & $U^{12}$ & $U^{13}$ & $U^{23}$ \\
\hline As1 & $0.00945(11)$ & $0.00745(10)$ & $0.00729(12)$ & $-0.00101(6)$ & $0.00113(7)$ & $0.00033(5)$ \\
O1 & $0.0173(6)$ & $0.0141(6)$ & $0.0086(6)$ & $0.0030(5)$ & $-0.0012(5)$ & $0.0016(4)$ \\
O2 & $0.0163(6)$ & $0.0169(6)$ & $0.0152(6)$ & $-0.0024(5)$ & $0.0046(5)$ & $-0.0073(5)$ \\
O3 & $0.0169(6)$ & $0.0074(5)$ & $0.0120(6)$ & $-0.0004(4)$ & $-0.0019(5)$ & $0.0021(4)$ \\
O4 & $0.0135(6)$ & $0.0175(6)$ & $0.0225(7)$ & $-0.0050(5)$ & $0.0016(5)$ & $0.0069(5)$
\end{tabular}


supporting information

\begin{tabular}{lllllll}
$\mathrm{C} 1$ & $0.0163(9)$ & $0.0145(8)$ & $0.0118(9)$ & $0.0017(6)$ & $0.0057(7)$ & $-0.0008(6)$ \\
$\mathrm{C} 2$ & $0.0147(8)$ & $0.0116(8)$ & $0.0161(9)$ & $-0.0020(6)$ & $0.0025(7)$ & $0.0008(6)$ \\
$\mathrm{N} 1$ & $0.0163(7)$ & $0.0109(7)$ & $0.0118(7)$ & $0.0020(6)$ & $-0.0023(6)$ & $-0.0022(5)$ \\
$\mathrm{N} 2$ & $0.0132(7)$ & $0.0087(6)$ & $0.0089(7)$ & $-0.0002(5)$ & $-0.0008(5)$ & $0.0004(5)$ \\
$\mathrm{C} 3$ & $0.0114(8)$ & $0.0133(8)$ & $0.0127(8)$ & $0.0019(6)$ & $0.0016(6)$ & $0.0009(6)$ \\
$\mathrm{C} 4$ & $0.0132(8)$ & $0.0118(8)$ & $0.0112(8)$ & $0.0021(6)$ & $0.0037(6)$ & $-0.0004(6)$ \\
$\mathrm{O} 5$ & $0.0142(6)$ & $0.0283(7)$ & $0.0149(7)$ & $-0.0046(6)$ & $0.0038(5)$ & $-0.0034(6)$ \\
\hline
\end{tabular}

Geometric parameters $\left(\AA,{ }^{\circ}\right)$

\begin{tabular}{|c|c|c|c|}
\hline As $1-\mathrm{O} 1$ & $1.6627(12)$ & $\mathrm{N} 1-\mathrm{H} 1 \mathrm{D}$ & 0.9200 \\
\hline $\mathrm{As} 1-\mathrm{O} 2$ & $1.6680(12)$ & $\mathrm{N} 2-\mathrm{C} 4$ & $1.482(2)$ \\
\hline $\mathrm{As} 1-\mathrm{O} 3$ & $1.6760(11)$ & $\mathrm{N} 2-\mathrm{C} 3$ & $1.485(2)$ \\
\hline As $1-\mathrm{O} 4$ & $1.7264(12)$ & $\mathrm{N} 2-\mathrm{H} 2 \mathrm{C}$ & 0.9200 \\
\hline $\mathrm{O} 4-\mathrm{H} 1$ & $0.842(10)$ & $\mathrm{N} 2-\mathrm{H} 2 \mathrm{D}$ & 0.9200 \\
\hline $\mathrm{C} 1-\mathrm{N} 1$ & $1.488(2)$ & $\mathrm{C} 3-\mathrm{C} 4^{\mathrm{ii}}$ & $1.517(2)$ \\
\hline $\mathrm{C} 1-\mathrm{C} 2^{\mathrm{i}}$ & $1.515(2)$ & $\mathrm{C} 3-\mathrm{H} 3 \mathrm{~A}$ & 0.9900 \\
\hline $\mathrm{C} 1-\mathrm{H} 1 \mathrm{~A}$ & 0.9900 & $\mathrm{C} 3-\mathrm{H} 3 \mathrm{~B}$ & 0.9900 \\
\hline $\mathrm{C} 1-\mathrm{H} 1 \mathrm{~B}$ & 0.9900 & $\mathrm{C} 4-\mathrm{C} 3^{\mathrm{ii}}$ & $1.517(2)$ \\
\hline $\mathrm{C} 2-\mathrm{N} 1$ & $1.487(2)$ & $\mathrm{C} 4-\mathrm{H} 4 \mathrm{~A}$ & 0.9900 \\
\hline $\mathrm{C} 2-\mathrm{C}{ }^{\mathrm{i}}$ & $1.515(2)$ & $\mathrm{C} 4-\mathrm{H} 4 \mathrm{~B}$ & 0.9900 \\
\hline $\mathrm{C} 2-\mathrm{H} 2 \mathrm{~A}$ & 0.9900 & $\mathrm{O} 5-\mathrm{H} 2$ & $0.842(10)$ \\
\hline $\mathrm{C} 2-\mathrm{H} 2 \mathrm{~B}$ & 0.9900 & $\mathrm{O} 5-\mathrm{H} 3$ & $0.836(10)$ \\
\hline $\mathrm{N} 1-\mathrm{H} 1 \mathrm{C}$ & 0.9200 & & \\
\hline $\mathrm{O} 1-\mathrm{As} 1-\mathrm{O} 2$ & $112.53(6)$ & $\mathrm{C} 2-\mathrm{N} 1-\mathrm{H} 1 \mathrm{D}$ & 109.2 \\
\hline $\mathrm{O} 1-\mathrm{As} 1-\mathrm{O} 3$ & $111.68(6)$ & $\mathrm{C} 1-\mathrm{N} 1-\mathrm{H} 1 \mathrm{D}$ & 109.2 \\
\hline $\mathrm{O} 2-\mathrm{As} 1-\mathrm{O} 3$ & $111.44(6)$ & $\mathrm{H} 1 \mathrm{C}-\mathrm{N} 1-\mathrm{H} 1 \mathrm{D}$ & 107.9 \\
\hline $\mathrm{O} 1-\mathrm{As} 1-\mathrm{O} 4$ & $105.86(6)$ & $\mathrm{C} 4-\mathrm{N} 2-\mathrm{C} 3$ & $111.79(12)$ \\
\hline $\mathrm{O} 2-\mathrm{As} 1-\mathrm{O} 4$ & $107.62(6)$ & $\mathrm{C} 4-\mathrm{N} 2-\mathrm{H} 2 \mathrm{C}$ & 109.3 \\
\hline $\mathrm{O} 3-\mathrm{As} 1-\mathrm{O} 4$ & $107.33(6)$ & $\mathrm{C} 3-\mathrm{N} 2-\mathrm{H} 2 \mathrm{C}$ & 109.3 \\
\hline $\mathrm{As} 1-\mathrm{O} 4-\mathrm{H} 1$ & $108.0(16)$ & $\mathrm{C} 4-\mathrm{N} 2-\mathrm{H} 2 \mathrm{D}$ & 109.3 \\
\hline $\mathrm{N} 1-\mathrm{C} 1-\mathrm{C} 2^{\mathrm{i}}$ & $110.60(13)$ & $\mathrm{C} 3-\mathrm{N} 2-\mathrm{H} 2 \mathrm{D}$ & 109.3 \\
\hline $\mathrm{N} 1-\mathrm{C} 1-\mathrm{H} 1 \mathrm{~A}$ & 109.5 & $\mathrm{H} 2 \mathrm{C}-\mathrm{N} 2-\mathrm{H} 2 \mathrm{D}$ & 107.9 \\
\hline $\mathrm{C} 2 \mathrm{i}-\mathrm{C} 1-\mathrm{H} 1 \mathrm{~A}$ & 109.5 & $\mathrm{~N} 2-\mathrm{C} 3-\mathrm{C} 4^{\mathrm{ii}}$ & $110.19(13)$ \\
\hline $\mathrm{N} 1-\mathrm{C} 1-\mathrm{H} 1 \mathrm{~B}$ & 109.5 & $\mathrm{~N} 2-\mathrm{C} 3-\mathrm{H} 3 \mathrm{~A}$ & 109.6 \\
\hline $\mathrm{C} 2^{\mathrm{i}}-\mathrm{C} 1-\mathrm{H} 1 \mathrm{~B}$ & 109.5 & $\mathrm{C} 4 \mathrm{ii}-\mathrm{C} 3-\mathrm{H} 3 \mathrm{~A}$ & 109.6 \\
\hline $\mathrm{H} 1 \mathrm{~A}-\mathrm{C} 1-\mathrm{H} 1 \mathrm{~B}$ & 108.1 & $\mathrm{~N} 2-\mathrm{C} 3-\mathrm{H} 3 \mathrm{~B}$ & 109.6 \\
\hline $\mathrm{N} 1-\mathrm{C} 2-\mathrm{C}^{\mathrm{i}}$ & $110.39(13)$ & $\mathrm{C} 4 \mathrm{ii}-\mathrm{C} 3-\mathrm{H} 3 \mathrm{~B}$ & 109.6 \\
\hline $\mathrm{N} 1-\mathrm{C} 2-\mathrm{H} 2 \mathrm{~A}$ & 109.6 & $\mathrm{H} 3 \mathrm{~A}-\mathrm{C} 3-\mathrm{H} 3 \mathrm{~B}$ & 108.1 \\
\hline $\mathrm{C} \mathrm{i}^{-}-\mathrm{C} 2-\mathrm{H} 2 \mathrm{~A}$ & 109.6 & $\mathrm{~N} 2-\mathrm{C} 4-\mathrm{C} 3^{\mathrm{ii}}$ & $110.66(13)$ \\
\hline $\mathrm{N} 1-\mathrm{C} 2-\mathrm{H} 2 \mathrm{~B}$ & 109.6 & $\mathrm{~N} 2-\mathrm{C} 4-\mathrm{H} 4 \mathrm{~A}$ & 109.5 \\
\hline $\mathrm{C}^{\mathrm{i}}-\mathrm{C} 2-\mathrm{H} 2 \mathrm{~B}$ & 109.6 & $\mathrm{C} 3^{\mathrm{ii}}-\mathrm{C} 4-\mathrm{H} 4 \mathrm{~A}$ & 109.5 \\
\hline $\mathrm{H} 2 \mathrm{~A}-\mathrm{C} 2-\mathrm{H} 2 \mathrm{~B}$ & 108.1 & $\mathrm{~N} 2-\mathrm{C} 4-\mathrm{H} 4 \mathrm{~B}$ & 109.5 \\
\hline $\mathrm{C} 2-\mathrm{N} 1-\mathrm{C} 1$ & $111.96(13)$ & 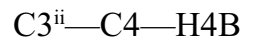 & 109.5 \\
\hline $\mathrm{C} 2-\mathrm{N} 1-\mathrm{H} 1 \mathrm{C}$ & 109.2 & $\mathrm{H} 4 \mathrm{~A}-\mathrm{C} 4-\mathrm{H} 4 \mathrm{~B}$ & 108.1 \\
\hline $\mathrm{C} 1-\mathrm{N} 1-\mathrm{H} 1 \mathrm{C}$ & 109.2 & $\mathrm{H} 2-\mathrm{O} 5-\mathrm{H} 3$ & $107(2)$ \\
\hline
\end{tabular}


supporting information

\begin{tabular}{|c|c|c|c|}
\hline $\mathrm{C} 1-\mathrm{C} 2-\mathrm{N} 1-\mathrm{C} 1$ & $-56.31(19)$ & $\mathrm{C} 4-\mathrm{N} 2-\mathrm{C} 3-\mathrm{C} 4^{\mathrm{ii}}$ & $-56.50(19)$ \\
\hline $\mathrm{C} 2 \mathrm{i}-\mathrm{C} 1-\mathrm{N} 1-\mathrm{C} 2$ & $56.43(19)$ & $\mathrm{C} 3-\mathrm{N} 2-\mathrm{C} 4-\mathrm{C}^{\mathrm{ii}}$ & $56.77(19)$ \\
\hline
\end{tabular}

Symmetry codes: (i) $-x,-y+1,-z+1$; (ii) $-x,-y+2,-z+1$.

Hydrogen-bond geometry $\left(A,{ }^{\circ}\right)$

\begin{tabular}{lllll}
\hline$D-\mathrm{H} \cdots A$ & $D-\mathrm{H}$ & $\mathrm{H} \cdots A$ & $D \cdots A$ & $D-\mathrm{H} \cdots A$ \\
\hline $\mathrm{O} 4-\mathrm{H} 1 \cdots \mathrm{O} 5^{\mathrm{iii}}$ & $0.84(1)$ & $1.81(1)$ & $2.6492(19)$ & $173(2)$ \\
$\mathrm{N} 1-\mathrm{H} 1 C \cdots \mathrm{O} 2$ & 0.92 & 1.73 & $2.6482(18)$ & 171 \\
$\mathrm{~N} 1-\mathrm{H} 1 D^{\cdots} \cdots 3^{\text {iv }}$ & 0.92 & 1.76 & $2.6722(18)$ & 173 \\
$\mathrm{~N} 2-\mathrm{H} 2 C \cdots \mathrm{O} 1^{v}$ & 0.92 & 1.77 & $2.6788(18)$ & 169 \\
$\mathrm{~N} 2-\mathrm{H} 2 D \cdots \mathrm{O} 3$ & 0.92 & 1.74 & $2.6548(18)$ & 172 \\
$\mathrm{O} 5-\mathrm{H} 2 \cdots \mathrm{O} 2$ & $0.84(1)$ & $1.86(1)$ & $2.6998(18)$ & $173(2)$ \\
$\mathrm{O} 5-\mathrm{H} 3 \cdots \mathrm{O} 1^{\text {vi }}$ & $0.84(1)$ & $1.89(1)$ & $2.7065(18)$ & $164(2)$
\end{tabular}

Symmetry codes: (iii) $x-1, y, z$; (iv) $-x+1 / 2, y-1 / 2,-z+3 / 2$; (v) $x-1 / 2,-y+3 / 2, z-1 / 2$; (vi) $x+1 / 2,-y+3 / 2, z-1 / 2$. 